Meta

Journal des traducteurs

Translators' Journal

\title{
Index des mots et sujets traités dans le volume 12
}

Volume 13, numéro 2, juin 1968

URI : https://id.erudit.org/iderudit/003218ar

DOI : https://doi.org/10.7202/003218ar

Aller au sommaire du numéro

\section{Éditeur(s)}

Les Presses de l'Université de Montréal

ISSN

0026-0452 (imprimé)

1492-1421 (numérique)

Découvrir la revue

Citer ce document

(1968). Index des mots et sujets traités dans le volume 12. Meta, 13(2), 95-102.

https://doi.org/10.7202/003218ar d'utilisation que vous pouvez consulter en ligne.

https://apropos.erudit.org/fr/usagers/politique-dutilisation/ 


\section{INDEX DES MOTS ET SUJETS TRAITES DANS LE VOLUME 12}

Abat-jour, 4: 112.

-able, 4: 114.

Acheter de l'assurance, 4: 134.

"Actif courant " , 4: 128 .

Actif disponible, 4: 128.

Actif réalisable à court terme, 4: 128.

Activités, 4: 114.

Adaptation, 4: 118.

Adjectifs composés des noms de points cardinaux, 4: 112 .

"Administration d'une convention ", 4: 138. Aéronautique, $1: 21$.

Affichage lumineux, 4: 125.

" Agenda », 3: 98

Agent (d'assurance), 4: 134 .

Agent de maîtrise, 4: 138.

"Agent de réclamation", 2: 52 .

Aggraver le risque, 4: 135.

Agronomie, 1: 27.

" Aide visuelle », 4: 133.

AIIC, 2: 47.

Air Canada, 4: 113.

Air-France, 4: 113.

"Ajustement ", 4: 138.

Aller vent arrière, $3: 80$.

Allocations-logement, 4: 113.

Aluminium, 1: 27.

Ambitionner, 4: 114 .

Américanolâtrie, $3: 95$.

"Ampexer ", 4: 121.

Angine, 4: 131 .

Anglicisme, 1: 27; 4: 111.

Anovulatoire, 4: 131.

Application d'une convention, 4: 138.

Apurement des comptes, 4: 138.

Arbitrage, 4: 139.

Arrêt, 3: 86.

Art culinaire, 1: 20.

Artère, 3: 86

Assemblées délibérantes, $3: 98$.

Assurabilité, 4: 135.

Assurable, 4: 135.

Assurance, 4: 133 .

Assurance-décès, 1: 17.

Assurance-maladie, 4: 113; 4: 138.

"Assurance-santé ", 4: 138.

Assurances sociales, 1: 16.

Assurance-vie, 1: 17.

"Assurance-vie industrielle », 4: 133.
"Assurance-vie ordinaire ", 4: 133.

Assuré, 4: 135.

Assureur, 4: 135.

Astronomie, 2: 62 .

Attaque à main armée, 1: 15.

Audio-visuel, 4: 122.

Auto-, 4: 112; 4: 115.

Autocensure, 4: 115 .

Autocritique, 4: 115.

Autocuiseur, 4: 115.

Autodéfense, 4: 115.

Autodétermination, 4: 115.

Autofinancement, 4: 115.

Auto-information, 4: 115.

"Automation ", 4: 138.

Automatique, $4: 113 ; 4: 138$.

Automédication, 4: 115 .

Autosatisfaction, 4: 115 .

"Avantages marginaux *, 4: 138.

Avantages sociaux, 4: 138.

Aviation, 2: 65 .

"Avis de réclamation ", 2: 54.

Avoir trait, 4: 138.

Ayant droit, 2: 55.

Bande dessinée, 4: 113.

"Barbier ", 4: 139.

Bâtiment, 1: 20;2:64.

Benbelliste, 4: 114 .

"Bénéfice ", 1: 17.

Berline, 4: 112.

Bibliographie (assurances), 4: 136.

Bibliothèque, 4: 114.

« Bien-être social », 4: 139.

Biennale de Québec, 4: 141; 4: 143.

Bloc-papier, 4: 123.

Blondin, 3: 85 .

Bois, 1: 20.

Boisson, 4: 139.

Bonimenteur, 4: 118 .

Bottillons, 3: 91.

Bottines, 3: 91.

Boutoir, 3: 97.

Branche populaire, 4: 133.

Brasserie, 2: 64.

"Breuvage », 4: 139.

Brouillage, 2: 51 .

Bruit d'aiguille, 2: 51.

Bruit de fond, 2: 51 . 
Bruit de surface, 2: 51 .

Bruits, 2: 50.

Bruits mécaniques, 2: 51.

Buvoter, 1: 14.

Cadrer, 4: 122.

Calculatrice électronique, 4: 125.

Calque, $4: 135 ; 4: 139$.

Calviniste, $4: 114$.

Caméra-stylo, 4: 120.

Canalisations électriques, $3: 87$.

Caniveau, 3: 87 .

Capacité, 3: 85 .

Carte perforée, $4: 113$.

Carter, 4: 112.

Cartothèque, 4: 114.

Cégétiste, 4: 115.

Centre d'intérêt, 4: 113.

Céphalgie, 3: 95.

Cerf-volant, 4: 112.

Changement de vitesse, $3: 77$.

Chauffe-bain, 4: 112 .

Chaussée glissante par temps humide, 3: 77.

Chauve-souris, 4: 112 .

Chemin de câbles, $3: 87$.

Chimie, 2: 62 .

Cinéaste, 4: 119.

Cinéma, 4: 118; 4: 123.

Cinéma d'essai, 4: 120.

Cinéma direct, $4: 120$.

Cinémascope, 4: 121.

Cinémathèque, $4: 114$.

Cinématoscope, 4: 121.

Cinéma total, 4: 120 .

Cinéphile, 4: 119.

Cinescoper, 4: 121.

Circonstance aggravante, $4: 135$.

Circuits force, $3: 87$.

Circuits lumière, $3: 87$.

Claque, 3: 91.

Climatiser/climatiseur, 4: 127.

Clouer au pilori, 4: 113 .

Co-, 4: 138.

Code de morphologie, 1: 27.

Code déontologique, 4: 131.

Coiffeur, 4: 139.

Collision sémantique, 1: 23.

Comité international des terminologies, 4: 124.

Commande, 4: 126.

Commande à distance, $3: 87$.

Commerce, 2: 64 .

Commerce extérieur, 3: 99.

Commission de révision du vocabulaire technique, 1: 27.

Communauté, 4: 116.

Communications de masse, $4: 122$.
Complaisance, 4: 115.

Composition des mots, 4: 112.

Comptabilité, 1: 17; 4: 127.

Comptabilité de caisse, $3: 88$.

Comptabilité d'exercice, $3: 88$.

Comptabilité électronique, 4: 128.

Comptes à recevoir, 4: 128.

"Comptes recevables », 4: 128.

"Computateur", 4: 125.

Conciliation, 4: 139.

Concrétisation des noms abstraits, 4: 114.

Conduits de filerie, 3: 87 .

Conseil international de la langue française, 4: 144 .

Consommation, $3: 84$.

Construction, 2: 64.

Contacts linguistiques, $3: 101$.

Contraceptif, 4: 131.

"Contrat sinistré », 2: 53 .

Contribuable, 4: 114 .

Contrôle, 4: 126.

Contrôler, 4: 116.

Coopérant, 4: 117.

Correspondance, 1: 22.

Cotisation, 1: 17 .

Coucou, 4: 112.

Coupable, 4: 114 .

Cousu main, 4: 113.

Couvre-chaussures, $3: 91$.

Critères terminologiques, $1: 23$.

Culture, 3: 75 .

Culture encyclopédique, 1: 29.

Curiosités, 4: 114.

Cyclisme, 1: 19.

De (introduisant un complément de matière), 2: 56 .

Débours, 3: 88 .

Déboursés, $3: 88$.

Décaissement, 3: 88 .

Déclaration de santé, 4: 135.

Déclaration (d'invalidité, d'accident), 2: 54.

Découvert de banque, 4: 128.

Défanatisation, 4: 115 .

Défanatiser, 4: 115.

Définition des tâches, $4: 138$.

Dé.ai de carence, 1: 17.

Demande de prestations, 2: 54 .

Demande de règlement, $2: 54$.

Demande d'indemnisation, 2: 54.

Demande d'indemnités, 2: 54.

Démarche, 4: 134.

Démercatoriser, 4: 115.

Démission, 4: 138.

Dénazification, 4: 115 .

Dénazifier, 4: 115.

"Département des réclamations », 2: 54. 
Dépenses, 3: 88.

Dépersonnalisation, 4: 115.

Dépersonnaliser, 4: 115.

Dépréciation, 1: 17.

Dérivation, 4: 111.

Dérivation des sigles, 4: 115.

Dérivation impropre, 4: 113.

Déstalinisation, $4: 115$.

Déterminé, 4: 138.

Deux-roues, 4: 113.

Devenir non assurable, 4: 135.

Diagramme, 3: 86 .

Diaphonie, 2: 51 .

Dictionnaires de langue, 1: 18.

Didactique, 4: 113.

Diffusion de l'information terminologique, 1: 25.

Diphasique, 4: 132.

Diplômé, 4: 138.

Diplôme de traduction, 4: 141 .

Diriger, 4: 118.

Dirigisme souple, $4: 144$.

Discothèque, 4: 114.

Discotrope, 4: 126.

"Discrimination ", 4: 138.

Distanciation, 4: 120.

Distinctions injustes, 4: 138.

Documentaliste, 4: 114 .

Documentation, 1: 25.

Documentation technique, $2: 60$.

Douane, 3: 99.

Droit, 1: 19;2:55;2:62.

Droit des peuples à disposer d'eux-mêmes, 4 : 115.

Droits d'auteur, 1: 6 .

Economie, 1: 20; 2: 63 .

Écran, 4: 120.

Ecrivasser, 1: 14.

Educationnel, 4: 114.

Efficacité (comme critère terminologique), 1: 15.

Électricité, 1: 21; 3: 85.

Electronique, 1: 19;1:21;2: 64 .

Electrotechnique, $1: 21 ; 2: 64$.

Eléments graphiques, 4: 133.

Eléments visuels, 4: 133.

Elever des prétentions, 2: 52 .

Emprunt, 1: 23; 3: 95; 3: 97; 4: 112; 4: 117; 4: 139.

En (avec complément de matière), 2: 56.

En circuit, 3: 86.

Enregistreur, 3: 86.

"Entraînement », 4: 138.

Entrée en jouissance, $1: 17$.

«Environnement », 3: 95.
Équipement des chantiers de construction, 1: 20.

-er, 4: 117.

"Escaler ", 4: 117.

-esque, 4: 120.

Essuie-mains, 4: 112.

Ethique professionnelle, 4: 141.

Eurafrique, 4: 113.

Eurailpass, 4: 113.

Eurasie, 4: 113.

Evolution, 4: 144.

Examens de traduction, 4: 140.

"Exhibit *, 4: 133.

Expert, 2: 52.

Expert-comptable, 4: 128.

Extemporané, 4: 132.

Extension sémantique, $4: 122$.

Faire du ski, 4: 117.

Faire souscrire, 4: 134.

Fardeau, 3: 84.

Fer-blanc, 3: 80.

Fétichisme littéraire, 4: 144.

Feu à éclat, 3: 86.

Feu à occultations, $3: 86$.

Feu clignotant, 3: 86.

Feu ondulant, $3: 86$.

Fiche, 4: 130.

Filerie, 3: 87.

Fleuriste, 4: 114.

Fonctionnel, 4: 114.

Formation, 4: 138.

Formation du traducteur, $1: 24 ; 1: 28$.

Formation gréco-latine, 4: 111.

Forme, 3: 95.

Forme (en), 4: 138.

" Formel ", 4: 138.

Fourrière, 3: 92.

France-Presse, 4: 113.

Francisation graphique, 3: 96.

Francisation orthographique, $3: 92$.

Francophonie, 4: 144.

Franglais, 4: 113.

Frou-frou, 4: 112.

Garantie, 1: 17.

Gaz, 4: 112 .

Générique, 4: 120.

Genre (utilisation fonctionnelle du), 1: 27.

Géographie, 1: 25; 1: 26.

Géologie, 2: 63.

Géomorphologie, 2: 63.

Gériatrie, 4: 139.

Glissement de sens, 3: 94.

"Gradué ", 4: 138.

Grammaire anglaise, 2: 66.

Grammaire structurale, 2: 58. 
Grande branche, 4: 133.

Grécomanie, 3: 95.

Groupement négociateur, 4: 138.

Haut pouvoir de coupure, 3: 86.

Hollywoodien, 4: 120 .

Honorable, 4: 114.

Hors circuit, $3: 86$.

Hybridation, 4: 115; 4: 126.

I (symbole), 3: 86 .

-ien, $4: 120$.

-ification, 4: 115 .

-ifier, 4: 115 .

Igamie, 4: 115.

Immobilisme linguisticque, 4: 111.

Impatiences, 3: 95.

Imperméable, 4: 114.

Impliquer, 4: 138.

Indemnité, 1: 17.

Inductance, $3: 86$.

Industrie-clé, 1: 14.

Informatique, 3: 99; 4: 125 .

Innovation, 4: 116.

Installation électrique, $3: 87$.

Intellectique, 3: 99.

Interaudiovision, 4: 123.

Interférence, 2: 51 .

Interférences linguistiques, 4: 133.

Interprétation, 2: 66 .

Interprète, $4: 127$.

Interpréter, $4: 127$.

Interpréteur, 4: 127.

Irlande du Nord, 4: 112.

-isation, 4: 115.

-iser, 4: 115.

-iste, 4: 114

Jargon, 1: 27.

Jetable, 4: 114; 4: 132.

« Joual », 1: 27.

Justification des prétentions, $2: 54$.

Juxtaposition (formation de néologismes), 4: 113 .

Kinématoscope, 4: 121.

Kinescope, 4: 121.

Kinétographe, $4: 121$.

Kinétoscope, 4: 121.

Landau, 4: 112.

Langage et pensée, 1: 24 .

Langage machine (en), 4: 126.

Langage parlementaire, 1: 21.

Langages documentaires, 2: 65 .

Langue de spécialité, 1: 27.

Latiniste, $4: 114$

Libéralisme linguistique, 4: 143.

Libre détermination, 4: 115 .

"Ligne de transmission ", 3: 87.

Ligne de transport, 3:87.
Linguistique et traduction, 2: 64 .

Linguistique structurale, $1: 7$.

Littéralisme, 1: 24.

Livre de messe, 3: 77.

Livres canadiens, 2: 60 .

Machine, 4: 126.

Machine à traduire, 2: 71 .

Magnétographe, 4: 121.

Magnétographier, 4: 121.

Magnétophonie, 4: 123.

Magnétoscope, 4: 121.

Mal-, 4: 115.

Maladie de Parkinson, 4: 112.

Mal de gorge, 4: 131.

Mal de Pott, 4: 112.

Mal de tête, 3: 95.

Mal d'oreille, 4: 131.

Malconfort, 4: 115.

Malentendant, 4: 115.

Malnutrition, 4: 115.

Malthusianisme linguistique, 4: 111.

Manuels, 4: 128.

Manutention, 2: 63 .

Marche, 3: 86.

Marge, 3: 92.

Maternage, 3: 90.

Mathématiques, 2: 63 .

Mécanique des roches, 2: 63 .

Mécanoïde, 4: 126.

Médecine, 3: 100.

Médiateurs techniques, 4: 123.

Médiation, 4: 139.

Médicaments brevetés, 4: 131.

Mémoire à accès sélectif, $4: 125$.

Message, 3: 75 .

Message météo, 4: 113.

Mesures, 3: 99.

Mesures de capacité, 3: 85 .

Métalloïde, 4: 126.

Méthode, 4: 138.

"Méthode de conférence ", 4: 139.

"Méthode des cas », 4: 139.

Mettre à (au) jour, 4: 113.

Milieu, 3: 95.

Miniaturisation, 4: 120.

"Minutes», 3: 98.

Mise en scène, $4: 120$.

Mondialvision, 4: 122.

"Mondiovision ", 4: 122.

Mondovision, 4: 122.

Montage, 4: 118.

Motivation des emprunts, $3: 95$.

Motivation des néologismes, 4: 126.

Motivation lexicale, 1: 15.

Motoculture, 4: 112. 
Mot-outil, 1: 14.

Mots composés, 1: 14.

Multifilaire, $3: 86$.

Naturaliste, $4: 114$.

-nel, 4: 114.

Néologisme, $1: 23 ; 3: 94 ; 3: 96 ; 4: 111 ; 4$ : 130.

Néologismes (création des), 4: 120.

Néo-réalisme, 4: 120 .

-ner, 4: 114

Nom propre employé comme nom commun, 4: 112 .

Non (emploi du trait d'union), 4: 138.

Nord-africain, 4: 112 .

"Nord Viêt-nam ", 4: 112.

Normalisation, 4: 121; 4: 126; 4: 137.

Normalisation de la langue technique, 1: 27.

Norme linguistique, 4: 144.

Normes professionnelles, 1: 6 .

Notable, 4: 114.

Nouveautés, $4: 114$.

O (symbole), 3: 86 .

Observation, 4: 116.

Observer, 4: 116 .

Officiel, 4: 138.

Omnipraticien, 4: 112.

On, 2: 39-44.

Onomatopée, 4: 112.

Onusien, 4: 115 .

Opéra, 3: 102.

Opérationnel, $4: 114$.

Opinion du risque, 4: 135.

Ordinateur, 4: 125.

Ordinateur et traducteurs, 2: 70.

Ordinolingue, 4: 126.

Ordre du jour, 3: 98 .

Otalgie, 4: 131 .

Ovationner, 4: 114.

"Paiement », 3:88.

Paiement de la réclamation, 2: 54.

Paiement des indemnités, 2: 54.

Paiement des prestations, 2: 54.

Paiement des sommes assurées, $2: 54$.

Panoramique, 4: 118; 4: 122.

Para-, 4: 115.

Parafiscalité, 4: 116.

Parajudiciaire, 4: 116.

Paramédical, 4: 116.

Paramilitaire, 4: 116.

Parapluie, 4: 116.

Parasite industriel, 2: 50.

Parasites, 2: 51.

Parasites atmosphériques, 2: 50.

Paratonnerre, 4: 116.

Parlers locaux, 3: 96.

Passif, 2: 40, 42.
Patron, 3: 95.

Pensée, 3: 75.

Pépier, 4: 112.

Percepteur, 4: 113.

Perforateur, 4: 127.

Perforeur, 4: 127.

Pharmacologie, 2: 62 .

Phénoménal, 3: 76.

Phonothèque, 4 : 114.

Phtonguème, 1: 15.

Physique, 4: 113.

Pièce de démonstration, 4: 133.

Pièces d'exposition, 4: 133.

Pinacothèque, 4: 114.

"Plan », 4: 122; 4: 138.

"Plan de pension », 2: 52 .

Plan-séquence, 4: 122.

Pléthore lexicale, 1: 27; 4: 125.

Poésie, 3: 100.

Poétique, 4: 113.

Poids et mesures, 2: 62 .

Points cardinaux dans les noms composés, 4: 112 .

Politique, 1: 20; 2: 63; 3: 100 .

Pommier, 1: 15.

Pompiste, 4: 114.

Position " couchette ", 4: 113.

Poste du bilan, 4: 128.

Poubelle, 4: 112.

Pouvoir de coupure, 3: 86 .

Pré-, 4: 115.

Préatomique, 4: 116.

Préavis, 4: 115 .

Précepteur, 4: 113.

Précinéma, 4: 118.

Précis, 4: 138.

Précolombien, 4: 116.

Préconstruction d'un film, 4: 119.

Précuisine, 4: 116.

Préemballé, 4: 116.

Préfabriqué, 4: 116.

Préfinancement, 4: 116.

Préfixation, $4: 113$.

Préindustriel, 4: 116.

Présence, 4: 120.

Prestation, 1: 17.

Prêt à porter, 4: 113 .

Prise en charge, 1: 17.

Problématique, 4: 113.

Procéder, 4: 116.

Procédure, 4: 116; 4: 138.

Procès-verbal, $3: 98$.

Producteur, 4: 118; 4: 134.

Profession, 4: 135.

Profondeur de champ, 4: 122. 
Programme, 4: 126.

Programmoïde, 4: 126.

Projections fixes, 4: 118.

Promotion, 4: 116.

Provignement, 4: 112.

Provignement sémantique, 4: 116.

Psychiatre, 4: 138.

Psychologie, 1: 19.

Puissance de précision, 3: 85 .

Puissance d'une génératrice, $3: 86$.

Purisme, 1: 27.

Pyralène, 3: 85 .

Qualités du traducteur, 1: 30 .

Racines scientifiques, 2: 61.

Radio, 3: 99.

Radioscopic, 1: 14.

Raisons sociales, 4: 113.

Rama, 4: 121.

Rassemblé, 3: 86.

Rattacher (se), 4: 138.

Réactance, 3: 86.

Réacteur, 3: 86.

Réadaptation, 4: 116.

Réaliser, 4: 116.

Réaliser (cinéma), 4: 118.

Réceptionner, 4: 114.

Recevoir, 4: 114.

"Réclamation admise ", 2: 53.

"Réclamation de décès », 2: 53.

«Réclamation payée », 2: 53.

Réclamation, réclamer, réclamant, 2: 51-55.

Reconnaissance des prétentions, 2: 54.

Rédaction, 1: 22.

Rédaction de rapports, 1: 22.

Rééducation, 4: 116.

"Référer », 4: 138.

Régime, 4: 138.

Régime de rente, 2: 52.

Règle à calcul, 3: 77 .

Relations industrielles, 4: 137.

Relief, 4: 120.

Remontée mécanique, 4: 113.

Remorquage, 3: 92.

"Rencontrer », 4: 139 .

Rente, 1: 17.

Réponse verbale, 4: 125.

Réservation, 4 : 116.

Réserver, 4: 116.

Responsabilités, 4: 114.

Reviseur, 4: 128.

Révolutionner, 4: 114.

Rigorisme linguistique, 4: 143.

Riocher, 2: 56.

Rioter, 2: 56.

Risque, 4: 135.
Robot, 2: 51.

"Routine ", 4: 126.

Rubrique, 4: 138.

Saisie, 3: 92.

Sangsue, 4: 112.

Sapidité, $4: 131$.

Satellite, 4: 116.

Scénariste, 4: 118.

Schéma, 3: 86.

Schéma de câblage, $3: 86$.

Schéma de circuits, $3: 86$.

Schéma de filerie, $3: 86$.

Schéma de réalisation, $3: 86$.

Schéma fonctionnel, 3: 86.

Sciences, 2: 65 .

Sciences infirmières, $3: 89$.

Sciences nucléaires, 2: 62.

Secouriste, 4: 114.

Secteur, 4: 116.

Sécurité sociale, 1: 21; 4: 139.

Semi-développé, 3: 86 .

Séminaires de traduction, 1: 6 .

Séparation des mots, 4: 138.

"Séparation volontaire », 4: 138.

Séquence, 4: 122.

Séquence progressive, 4: 112.

Séquence régressive, 4: 112.

Séquentiel, 4: 132.

Séries analogiques, 3: 96.

Service des règlements, $2: 54$.

Service des réservations, 4: 116.

Service des sinistres, $2: 54$.

Sigle, 4: 114

Sinistre, $1: 17 ; 2: 53$.

Sinistre mortuaire, 2: 53.

Sinistre reconnu, 2: 53.

Sinistre réglé, 2: 53.

Skier, 4: 117.

Société française des traducteurs, 2: 70

Soignance, 3: 90.

Soins à domicile, $3: 95$.

Soins infirmiers, $3: 88$.

Solutionner, 4: 114.

Sourd, 4: 115 .

Sous-alimentation, $4: 115$.

Souscrire, 4: 134.

Sous-programme, 4: 126.

"Soutiré de banque ", 4: 128.

Spécialités pharmaceutiques, 4: 131.

"Spécification des tâches », 4: 138.

"Spécifique ", 4: 138.

Stage, 1: 31 .

«Statique », 2: 50.

Statut du traducteur, 1: 6 .

Stéréophonie, 4: 120.

Studio, 4: 118. 
Style, 1: 18.

Substantivation des adjectifs, 4: 113.

Sud-américain, 4: 112.

Suffixation, 4: 113 .

Suffixe de profession, 4: 114.

Super, 4: 121.

"Superviseur ", 4: 138.

Survenance, 2: 53 .

Symbolique, 4: 113.

Syntagme, 4: 113.

Tableau de feutre, 4: 123.

Tableau magnétique, $4: 123$.

-té, 4: 114.

Télécommande, $3: 87$.

Télécommunications, $1: 21 ; 2: 64$.

Téléphone, 4: 112.

Télescopage, 4: 113 .

Télétraitement, 4: 125 .

Tendances analytiques, $3: 77$.

Termes bancaires, $1: 20 ; 1: 21$.

Termes de médecine, 3: 100.

Termes juridiques, 4: 134 .

Termes médico-hospitaliers, 3: 97.

Termes pharmaceutiques, 4: 130.

Termes techniques, $1: 21$.

Terminologie, 1: 23-28.

Terminologie comptable, 1: 17; 4: 127.

Terminologie pharmaceutique, $1: 25 ; 4: 130$.

Théâtre d'opérations, 4: 120.

Théorie de la traduction, $1: 3,4 ; 1: 21$.

Théorie et pratique, 1: 30 .

-thèque, $4: 114$.

Thermodynamique, $4: 112$.

Thyratron, 3: 87 .

Thyristor, $3: 87$.

Timbre-poste, 4: 113.

-tion, 4: 114.

Titre, 2: 55 .

"Titre-sujet », 4: 138.

Titulaire, 1: 17.

Topographie, 3: 86 .
Tour de manivelle, 4: 120.

Tourner, 4: 117.

Tout compris, 4: 113.

Traduction automatique, 1: 4, 7; 3: 103.

Traduction et terminologie, 1: 24

Traduction littéraire, 1: 4.

Traduction publicitaire, 3: 79 .

Traitement en direct, 4: 125.

Traitement en parallèle, $4: 125$.

Trampolin, 3: 92.

Tranquillisant, 4: 113.

Transcripteur, 2: 51 .

Transfusion sanguine, 1: 25.

Transistor, 3: 87.

Transmission, 3: 87 .

Transport, 3: 87.

Transport à câble, 2: 64 .

Travaux en cours, $3: 77$.

Travaux publics, $1: 20 ; 2: 64$.

Tréfilerie, $3: 87$.

Tremplin, 3: 92.

Trempolin, 3: 92.

Triphasique, $4: 132$

Unifilaire, $3: 86$.

Uniservice, 4: 132.

"Unité de négociation ", 4: 138.

URSS (traduction en), 1: 3 .

Utilisation fonctionnelle du genre, 4: 127.

"Vendre de l'assurance ", 4: 134.

Vérification/vérificateur, $4: 127$.

Vérifieur, 4: 127.

Viêt-nam du Nord, 4: 112

Vinaigre, $4: 112$.

Vitagraphe, 4: 121 .

Vitascope, 4: 121 .

Vocabulaire commercial, 3: 99.

Vocabulaire scientifique, $3: 94$.

Voiture tous terrains, $4: 113$.

Voix passive, 2: 40.

Voyelle thématique, 1: 27 .

Zone de stationnement interdit, 3: 77. 


\section{INDEX OF WORDS AND TOPICS IN VOLUME 12}

Abbreviations, 2: 61.

Accrual accounting, 3: 88 .

Added risk, 4: 135.

Admission of claim, 2: 54.

Advertising, 2: 65 .

Aeronautical terms, 2: 64 .

Affect (to) insurability, 4: 135.

American language, 2: 61 .

Angledozer, 3: 97.

Appraise (to) insurability, 4: 135.

Architecture, 1: 19; 1: 20.

As, 1: 16.

Askare1, 3: 85 .

Assembled, 3: 86 .

Astronomy, 1: 20.

Australian English, 3: 100.

Automatic translation, 2: 65 .

Back-ground noises, 2: 51.

Bathing beauty, 4: 119.

Bilingualism, 2: 60; 4: 141 .

Bivalence of an interpreter, 2: 46.

Block-booking, 4: 119.

Block diagram, $3: 86$.

Block-system, 4: 119.

Brainwashing, 4: 139.

Bulldozer, 3: 97.

Burden, 3: 85 .

Business, 2: 62 .

Buy (to) insurance, 4: 134.

Cableway, 3: 85.

Cabling diagram, $3: 86$.

Calf dozer, 3: 97.

Can, 1: 22.

Canadian English, 3: 98.

Capacitance, 3: 85 .

Cash accounting, $3: 88$.

Cast, 4: 119

Center, 4: 127.

Centre, 4: 127.

Chart, 3: 86.

Chemical technology, 1: 21.

Chemistry, 1: 21.

Circuit diagram, 3: 86.

Claim, 2: 51-55.

Claim admitted, 2: 53.

Claimant, 2: 55

Claim paid, 2: 53 .

Claims department, 2: 54.

Close shot, 4: 119.

Commercial phrases, 2: 64.
Commercial translation, $3: 81$.

Computer, 1: 20;3: 99 .

Connection diagram, $3: 86$.

Continuity, 4: 119.

Control system, 1: 20 .

Cosmetics, 2: 63 .

Cross-talk, 2: 51 .

Cuisine, 1: 20.

Current-limiting reactor, $3: 87$.

Dash-pot, 3: 96.

Data processing, 3: 99.

Death claim, 2: 53 .

Demand (to), 2: 52 .

Depreciation, 1: 17 .

Detergents, 2: 63.

Director, 4: 119.

Disbursement, 3: 88 .

Disc oriented, 4: 126.

Disposable, 4: 132.

Double overhead camshaft, 2: 60 .

Drink, 1: 19.

Economics, 1: 19; 2: 62 .

Educational, $4: 114$

Electrical engineering, 1: 19;2: 62 .

Electricity, 1: 20.

Electric mixer, 2: 51.

Electronics, 1: 19; 1: 20; 3: 99.

Elementary diagram, 3: 86 .

Ethical code, 4: 131 .

Ethical drug, 4: 131 .

Etymology, 1: 19; 2: 61.

Evidence of insurability, 4: 135.

Fan, 4: 119.

Feedback, 3: 99.

Feeder, 3: 86.

Financial terms, 2: 64.

Flash, 4: 119.

Flash-back, 4: 119.

Flasher, 3: 86.

Fly (to), 3: 76 .

Forestry, 2: 64.

Gag, 4: 119.

Gagman, 4: 119.

Gear shift, 3: 77.

General linguistics, 2: 65 .

Geography, 1: 19; 2: 63.

Giggle (to), 2: 56.

Grit, 3: 99.

Grounding transformer, 3:87.

Happening, 2: 53 . 